\title{
Particle production and energy deposition studies for the neutrino factory target station
}

\author{
John J. Back* \\ University of Warwick, Coventry, CV4 7AL, United Kingdom \\ Chris Densham \\ STFC Rutherford Appleton Laboratory, Didcot, OX11 OQX, United Kingdom \\ Rob Edgecock \\ STFC Rutherford Appleton Laboratory, Didcot, OX11 0QX, United Kingdom, \\ and Huddersfield University, Huddersfield, HD1 3DH, United Kingdom \\ Gersende Prior \\ European Organization for Nuclear Research, CERN CH-1211, Genève 23, Switzerland
}

(Received 19 October 2012; published 1 February 2013)

\begin{abstract}
We present FLUKA and MARS simulation studies of the pion production and energy deposition in the Neutrino Factory baseline target station, which consists of a 4 MW proton beam interacting with a liquid mercury jet target within a $20 \mathrm{~T}$ solenoidal magnetic field. We show that a substantial increase in the shielding is needed to protect the superconducting coils from too much energy deposition. Investigations reveal that it is possible to reduce the magnetic field in the solenoid capture system without adversely affecting the pion production efficiency. We show estimates of the amount of concrete shielding that will be required to protect the environment from the high radiation doses generated by the target station facility. We also present yield and energy deposition results for alternative targets: gallium liquid jet, tungsten powder jet, and solid tungsten bars.
\end{abstract}

DOI: 10.1103/PhysRevSTAB.16.021001

\section{INTRODUCTION}

The current baseline option for the Neutrino Factory [1] is to use a $4 \mathrm{MW}$ proton beam interacting with a freeflowing mercury jet to create an intense muon beam [2]. The MERIT experiment has shown a proof-of-principle demonstration of a high intensity liquid mercury jet target [3]. The interaction of the bunched proton beam (rms bunch length equal to $3 \mathrm{~ns}$ ) with the mercury jet creates low-energy pions that are captured by the high field $(\sim 20 \mathrm{~T})$ solenoid and transported through a decay channel. Muons resulting from the decay of these pions pass through a cooling section and circulate around a storage ring until they decay to neutrinos.

In this paper, we present a series of simulation studies, using the FLUKA [4] and MARS [5] computer packages, on particle production and energy deposition (radiation dose) calculations. We first show the simulation results for the so-called Study 2 geometry configuration, and then describe how the geometry needs to be modified to address various safety issues. We also compare useful muon yields and energy deposition doses for different target material

\footnotetext{
*J.J.Back@warwick.ac.uk
}

Published by the American Physical Society under the terms of the Creative Commons Attribution 3.0 License. Further distribution of this work must maintain attribution to the author(s) and the published article's title, journal citation, and DOI.
PACS numbers: 13.20.Cz, 13.75.Cs, 14.60.Ef, 29.25.-t

alternatives. Finally, we present a study on the concrete shielding requirements that will be necessary to protect the environment from the high radiation doses emanating from the target station.

\section{SIMULATION PARAMETERS}

As a starting point, the Neutrino Factory mercury jet target station geometry is based on the Study 2 configuration [6], as shown in Fig. 1, with the appropriate $20 \mathrm{~T}$ field

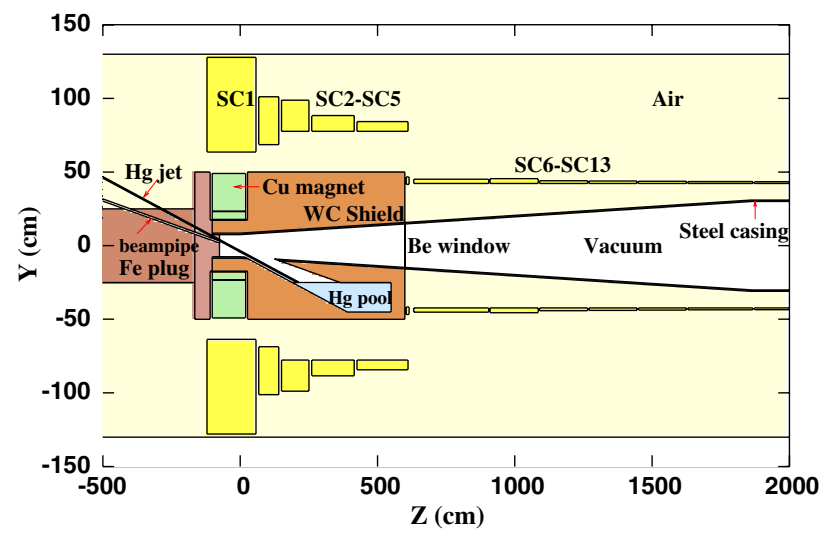

FIG. 1. Schematic of the Study 2 geometry of the Neutrino Factory target system. The shielding is comprised of tungsten carbide (80\%) with water cooling $(20 \%)$. The superconducting magnets are labeled $\mathrm{SC} n$, where $n=1$ to 13 . 
map based on the dimensions and currents in the normal-conducting copper $(\sim 6 \mathrm{~T})$ and superconducting $(\sim 14 \mathrm{~T})$ coils. Variations to the geometry are made to reduce the energy deposition in the superconducting magnets, as well as incorporating more engineering considerations to the overall target station design. In all simulations, the proton beam has a transverse Gaussian profile with a root mean square radius of $1.2 \mathrm{~mm}$. The kinetic energy of the proton beam is nominally set to $8 \mathrm{GeV}$, but is varied when finding the optimal number of useful muons from the target. The mercury jet is modeled as a simple cylinder with a radius of $4 \mathrm{~mm}$, tilted at approximately $100 \mathrm{mrad}$ to the magnetic $z$ axis. We also investigate the yields and energy deposition for alternative targets, also tilted at $100 \mathrm{mrad}$ to the $z$ axis: liquid gallium jet, powder tungsten jet (50\% density), and solid tungsten bars. The first two alternatives are also modeled as cylinders with a radius of $4 \mathrm{~mm}$, while the solid target is modeled as a $20 \mathrm{~cm}$ long, $2 \mathrm{~cm}$ diameter cylinder with a density of $19.25 \mathrm{~g} / \mathrm{cc}$. The angle between the target and the proton beam at their intersection $(z=-37.5 \mathrm{~cm})$ varies between 20 and $30 \mathrm{mrad}$, depending on the initial kinetic energy, in order to optimize pion production [7].

\section{STUDY 2 GEOMETRY}

\section{A. Muon yields}

One important figure of merit concerning the performance of the target is the total number of muons that pass through the Neutrino Factory cooling channel. This is calculated by first counting the number of pions, kaons, and muons that are directly produced by the mercury

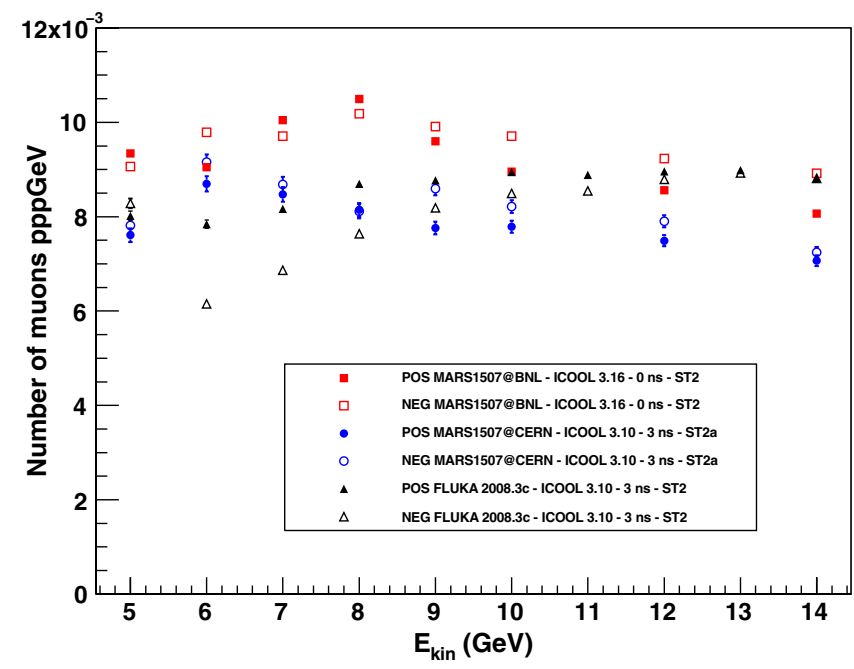

FIG. 2. The accepted muon yield per proton per $\mathrm{GeV}$ as a function of proton beam kinetic energy. The different points show what effect different simulation code versions have on the yield calculation results. ST2a and ST2 denote different $\underline{B}$ field tapering parameters. For ST2a (ST2), $|\underline{B}|$ adiabatically decreases from 20 to $1.75 \mathrm{~T}(1.25 \mathrm{~T})$ over a length of $12 \mathrm{~m}(18 \mathrm{~m})$. jet-proton beam interaction. These secondary particles are then tracked through the solenoidal target decay channel up to $z=50 \mathrm{~m}$. The ICOOL simulation package [8] is used to find what fraction of these secondary particles end up as muons within the accelerator acceptance $(30 \mathrm{~mm}$ transversely and $150 \mathrm{~mm}$ longitudinally, with $z$ momenta between 100 and $300 \mathrm{MeV} / \mathrm{c}$ ).

Figure 2 shows the expected muon yields for the Study 2 (ST2) and Study 2a (ST2a) target configurations, as a function of the kinetic energy of the incoming proton beam. These yields are normalized to the number of protons on target as well as the proton beam kinetic energy. In general, the results are in agreement with the modelindependent conclusions of Ref. [9] that "the dependence of the muon yield on proton beam energy at constant beam power is relatively flat, and any energy between 4 and $11 \mathrm{GeV}$ has a yield that is within $10 \%$ of the maximum at $7 \mathrm{GeV}$." There is some variation in the calculated muon yield, depending on what version of the simulation codes are used, as well as on the assumptions made about the proton beam bunch spacing ( 0 or $3 \mathrm{~ns}$ ). For the former, this can only be improved by updates to the simulation codes.

\section{B. Energy deposition}

Figure 3 shows the distribution of energy deposition in the Study 2 target station using the FLUKA simulation code, which agrees rather well with those results obtained using MARS [10]. Also shown is the total power dissipated in the first few superconducting coils. The values in SC1-SC3 are unacceptably high and certainly have to be reduced by increasing the shielding thickness [11]. Previous studies have shown that the forces between the superconducting coils are very high [12], and in the present geometry there is no space for support structures between individual coils, which needs to be addressed. Another issue is that the normal conducting magnets also experience very high

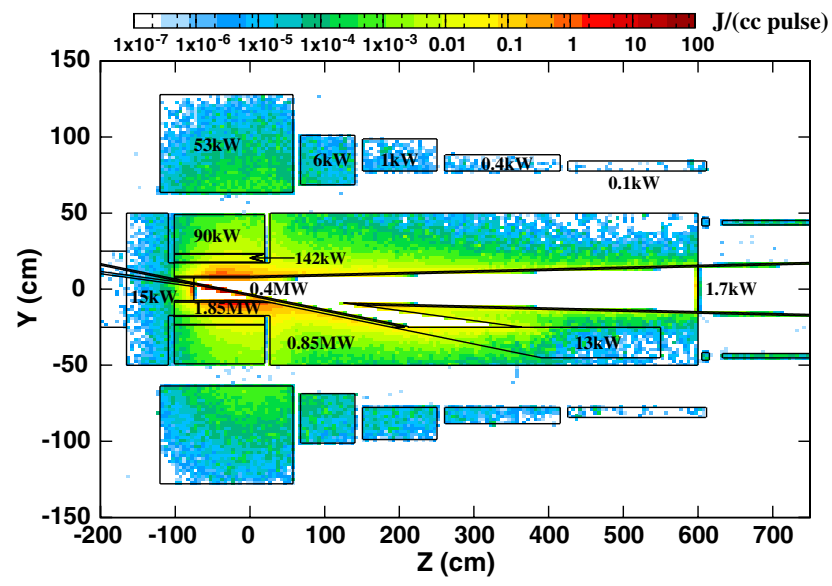

FIG. 3. Distribution of the deposited energy density (J/cc) per beam pulse ( $50 \mathrm{~Hz}$ repetition rate) in the Study 2 target system. Also shown is the estimated deposited power within various regions. 
levels of power dissipation (142 and $90 \mathrm{~kW}$ ). This problem is avoided by removing them completely and replacing the volume with more shielding. This means that the magnetic field in the beam-jet interaction region is decreased from $20 \mathrm{~T}$ down to $15 \mathrm{~T}$. Also note that the power deposition in the mercury pool is quite low $(13 \mathrm{~kW})$. This means that the volume of the pool reservoir needs to be increased in order for it to act as an effective beam dump for protons that do not interact with the mercury jet. In the next section we explore what effect these necessary geometry changes have on both the distribution of the energy deposition and on the accepted muon yield efficiencies.

\section{IMPROVED SHIELDING CONFIGURATION}

Figure 4 shows the new target station geometry configuration that has increased shielding (approximately doubled in thickness laterally) to protect the superconducting (SC) coils, with the normal conducting magnets removed, as well as a larger mercury pool reservoir $(88 \mathrm{~cm}<z<$ $367 \mathrm{~cm}, y<-15 \mathrm{~cm}, r<45 \mathrm{~cm}$ ). Table I provides the geometrical and current density parameters of the SC coils. The SC coils are arranged in triplets, each corresponding to one cryostat module. Gaps are introduced between neighboring coils to provide space for the cryostat modules and supporting structures to hold them in place (the cooling components and their internal shielding are not included in the simulation). Each cryostat is protected by large shielding volumes, made from tungsten beads (60\%) with an assumed low-grade density of $15.8 \mathrm{~g} / \mathrm{cc}$, and fast-flowing helium gas (40\%), which replaces water as the cooling agent. This change is motivated by the concern that any creation of bubbles in the water will affect its circulation, giving nonuniform cooling of the tungsten beads, as well as the problem of corrosion in a high radiation environment [13]. Each shielding section, separated by $20 \mathrm{~cm}$ gaps, is surrounded by stainless steel container vessels (each with a thickness between 2 and $10 \mathrm{~cm}$ ) that must support the

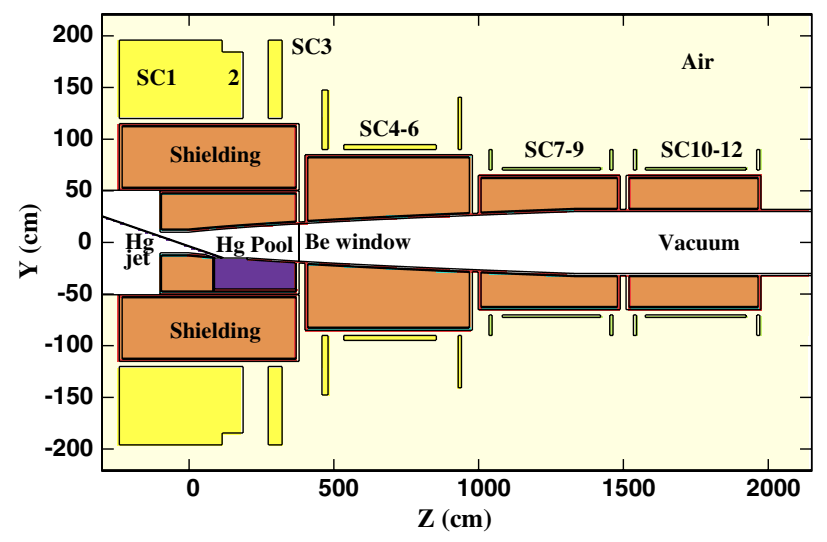

FIG. 4. Schematic of the updated target geometry configuration with increased shielding. The superconducting magnets are labeled SC $n$, where $n=1$ to 12 .
TABLE I. Parameters defining the superconducting (SC) coils for the new shielding geometry shown in Fig. 4: $z_{0}$ is the initial $z$ position, $\Delta z$ specifies the length along $z, r_{1}$ is the inner radius, $\Delta r$ is the radial thickness, while $I$ is the average current density. The last column specifies the coil materials used in the FLUKA simulation, where SCon is the compound $\mathrm{Cu}(54 \%), \mathrm{Nb}(24 \%)$, $\mathrm{Ti}(12 \%)$, and kapton (10\%). The mass densities for $\mathrm{Nb}_{3} \mathrm{Sn}$ and SCon are $6.8 \mathrm{~g} / \mathrm{cc}$ and $7.0 \mathrm{~g} / \mathrm{cc}$, respectively.

\begin{tabular}{|c|c|c|c|c|c|c|}
\hline Coil & $z_{0}(\mathrm{~cm})$ & $\Delta z(\mathrm{~cm})$ & $r_{1}(\mathrm{~cm})$ & $\Delta r(\mathrm{~cm})$ & $I\left(A / \mathrm{mm}^{2}\right)$ & Material \\
\hline SC1 & -240.5 & 355.0 & 120.0 & 75.8 & 19.3 & $\mathrm{Nb}_{3} \mathrm{Sn}$ \\
\hline $\mathrm{SC} 2$ & 114.5 & 72.7 & 120.0 & 64.3 & 22.0 & $\mathrm{Nb}_{3} \mathrm{Sn}$ \\
\hline SC3 & 273.6 & 48.1 & 120.0 & 75.8 & 26.7 & $\mathrm{Nb}_{3} \mathrm{Sn}$ \\
\hline $\mathrm{SC} 4$ & 459.0 & 21.3 & 90.0 & 57.6 & 33.8 & $\mathrm{Nb}_{3} \mathrm{Sn}$ \\
\hline SC5 & 534.6 & 319.7 & 90.0 & 4.7 & 40.9 & $\mathrm{Nb}_{3} \mathrm{Sn}$ \\
\hline SC6 & 929.8 & 11.2 & 90.0 & 50.6 & 41.9 & $\mathrm{Nb}_{3} \mathrm{Sn}$ \\
\hline SC7 & 1036.0 & 10.7 & 70.0 & 20.0 & 45.0 & SCon \\
\hline SC8 & 1081.7 & 339.5 & 70.0 & 2.5 & 46.7 & SCon \\
\hline SC9 & 1453.0 & 11.0 & 70.0 & 20.0 & 46.3 & SCon \\
\hline SC10 & 1534.7 & 10.7 & 70.0 & 20.0 & 45.8 & SCon \\
\hline SC11 & 1575.8 & 348.3 & 70.0 & 2.5 & 47.7 & SCon \\
\hline SC12 & 1960.3 & 11.0 & 70.0 & 20.0 & 45.8 & SCon \\
\hline
\end{tabular}

200 tonne weight while limiting stresses and deformations to acceptable values [14].

The beam pipe is modeled as a $2 \mathrm{~cm}$-thick stainless steel tapered volume, which defines the inner bore of the decay vacuum region. The beam pipe section just above the mercury pool surface is removed between $z=88$ and $200 \mathrm{~cm}$ to allow the mercury jet (and noninteracting protons) to enter the pool unhindered. The beryllium window, which separates the jet-beam interaction region from the rest of the decay channel vacuum, is moved further upstream to coincide with the edge of the first cryostat module. It consists of a $0.5 \mathrm{~cm}$ gap containing rapidly flowing $\mathrm{He}$ gas for cooling sandwiched between two $0.5 \mathrm{~cm}$ Be layers. The iron yoke plug has been removed to allow for space for the mercury jet nozzle injection and return flow system (which is ignored in the simulation). The mercury jet is still modeled as a simple cylinder of radius $4 \mathrm{~mm}$, tilted at approximately $100 \mathrm{mrad}$ with respect to the magnetic $z$ axis.

\section{A. Pion and muon yields}

The removal of the normal conducting magnets implies that the peak magnetic field in the jet-beam interaction region is decreased from 20 to $15 \mathrm{~T}$. To ensure that secondary pions from the target maintain their magnetic rigidity, the inner bore radius of the beam pipe at this location is increased from 7.5 to $10 \mathrm{~cm}$. Figure 5 shows a comparison of the axial magnetic field profile between the original Study 2 geometry and the new increased shielding geometry. The latter has a much wider magnetic field profile, which helps to capture much more secondary charged particles from the target. This can be clearly 


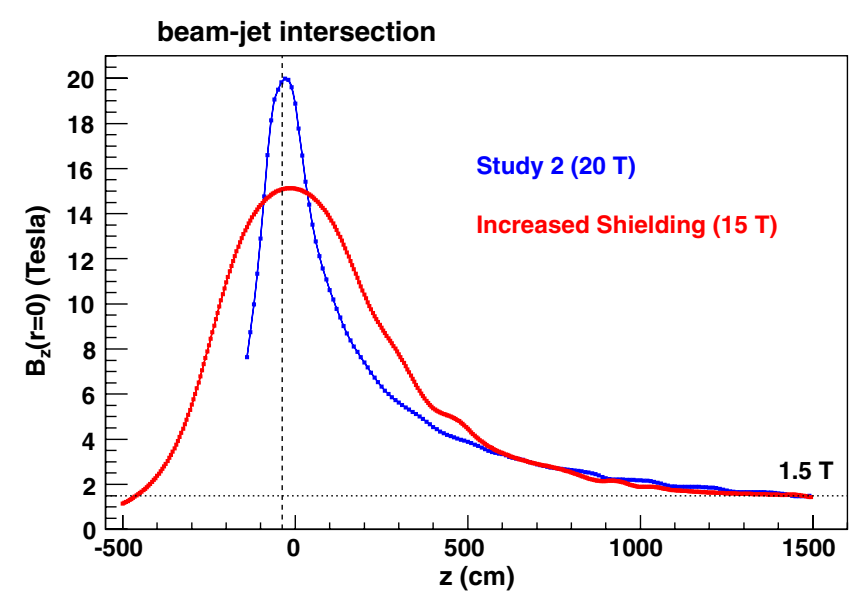

FIG. 5. Axial magnetic field profile distributions for the Study 2 and new increased shielding geometries.

seen in Fig. 6, which shows the improved chargedaveraged yield of useful pions and muons from the target, normalized to the total number of protons on target and the initial beam kinetic energy. Using the FLUKA simulation package, these yields are calculated by finding the number of pions and muons (of both signs) that pass a transverse plane $50 \mathrm{~m}$ downstream from the beam-jet interaction region, within the decay channel aperture that has a bore radius of $30 \mathrm{~cm}$. To obtain a figure of merit for the muon yield for the Neutrino Factory, we require that these particles have kinetic energies between 40 and $180 \mathrm{MeV}$ [7].

In addition to the baseline mercury jet case, the yields from alternative target materials are also shown in Fig. 6, using the simulation parameters outlined in Sec. II. Between 5 and $15 \mathrm{GeV}$, there is a two-peak structure in the normalized yield distributions, owing to the transition between different hadronic models used at low and high

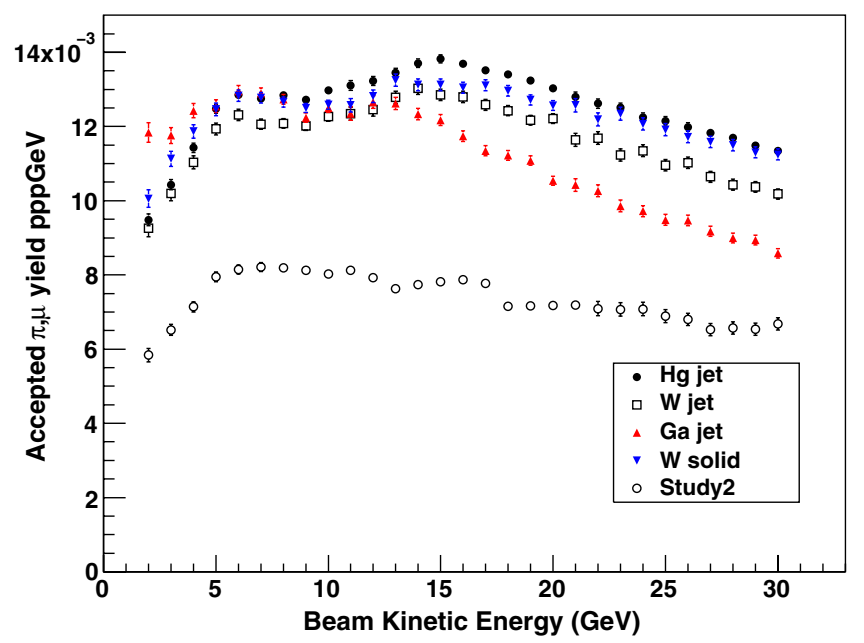

FIG. 6. The charged-averaged accepted pion and muon yield per proton per $\mathrm{GeV}$ for various targets in the new increased shielding geometry. Also shown are the equivalent yields for the mercury jet target in the Study 2 geometry. energies in the FLUKA simulation code. Above $5 \mathrm{GeV}$, the mercury jet provides the best yields, although the performance of the solid tungsten target matches this very closely. Note that we do not see a dramatic reduction in the yield for the tungsten powder jet, which is assumed to have an effective density of $50 \%$ of solid tungsten. The reason for this is that, even though less protons will interact with the powder target, the amount of reabsorption of secondary particles inside the target will also be lower, giving an overall figure of merit comparable to either the mercury jet or solid tungsten case. At low beam kinetic energy (below $5 \mathrm{GeV}$ ), there is an indication that the liquid gallium target gives the best yield compared to the other materials. Further analysis is required to optimize the yields for this improved shielding geometry.

\section{B. Energy deposition}

We have seen that the change to the target station geometry has had a positive effect to the useful muon yield. The situation regarding the energy deposition for the superconducting coils is also improved. Figure 7 shows the energy deposition in the new target station geometry, obtained using the FLUKA simulation code, which shows that the increase to the shielding has dramatically reduced the power dissipation in the SC coils. Table II provides a detailed breakdown of the deposited power in various subregions of the new geometry, where the uncertainties are estimated by using different initial random number seeds for the simulation. The first SC coil experiences a total power of $0.4 \mathrm{~kW}$, with the other coils experiencing much lower values, giving a total approximately equal to $0.6 \mathrm{~kW}$. These correspond to energy densities below $0.1 \mathrm{~mW} / \mathrm{g}$, which permits at least a 10 year (of $2 \times 10^{7} \mathrm{~s}$ each) operational lifetime against radiation damage to their

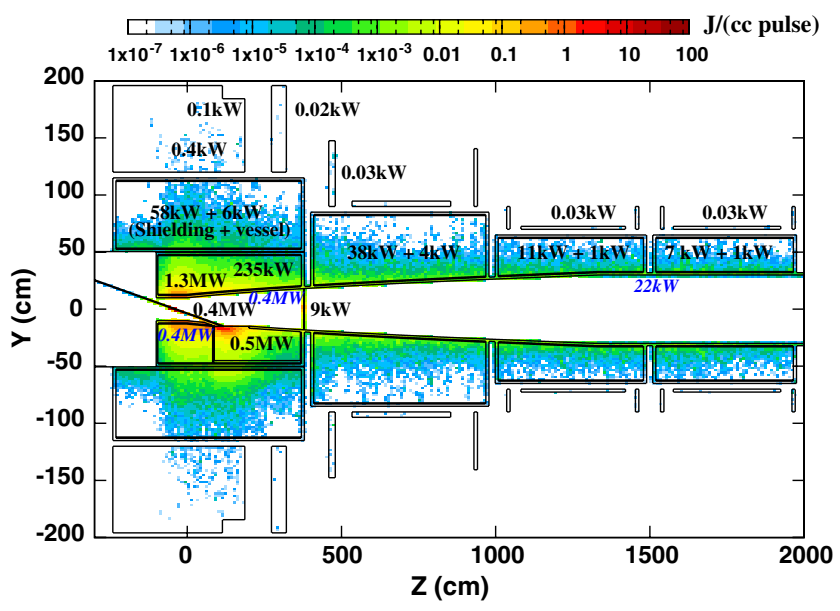

FIG. 7. Distribution of the deposited energy density (J/cc) per beam pulse $(50 \mathrm{~Hz}$ repetition rate) in the improved shielding geometry configuration for the mercury jet target. Also shown is the estimated power deposition within various regions (blue italics denote the power deposited in the beam pipe). 
TABLE II. Power deposition in various regions of the increased shielding geometry configuration for the mercury jet, as well as for alternative target materials. For the solid tungsten bars, the pool reservoir is replaced by more shielding.

\begin{tabular}{|c|c|c|c|c|}
\hline \multirow[b]{2}{*}{ Region } & \multicolumn{4}{|c|}{ Deposited power $(\mathrm{kW})$} \\
\hline & $\mathrm{Hg}$ jet & Ga jet & W powder jet & W solid \\
\hline SC coils $1-12$ & $0.57 \pm 0.05$ & $0.67 \pm 0.06$ & $0.62 \pm 0.06$ & $0.55 \pm 0.06$ \\
\hline Lower shielding SC $1-3(r<50 \mathrm{~cm}, z<83 \mathrm{~cm})$ & $1284.4 \pm 8.3$ & $1034.9 \pm 8.1$ & $1154.3 \pm 9.0$ & $1282.6 \pm 7.1$ \\
\hline Lower shielding SC $1-3(r<50 \mathrm{~cm}, z>83 \mathrm{~cm})$ & $234.2 \pm 3.5$ & $318.5 \pm 3.6$ & $284.8 \pm 4.0$ & $348.2 \pm 6.2$ \\
\hline Upper shielding SC 1-3 $(r>50 \mathrm{~cm})$ & $58.3 \pm 0.8$ & $82.6 \pm 1.1$ & $75.8 \pm 1.0$ & $41.9 \pm 0.5$ \\
\hline Shielding for SC 4-6 & $38.0 \pm 1.7$ & $45.2 \pm 2.0$ & $38.8 \pm 1.9$ & $26.1 \pm 1.2$ \\
\hline Shielding for SC 7-9 & $11.0 \pm 0.7$ & $11.9 \pm 0.8$ & $10.8 \pm 0.8$ & $8.1 \pm 0.6$ \\
\hline Shielding for SC $10-12$ & $7.4 \pm 0.7$ & $8.1 \pm 0.7$ & $7.1 \pm 0.8$ & $5.0 \pm 0.5$ \\
\hline Beam pipe up to $z=0 \mathrm{~cm}$ & $352.3 \pm 2.9$ & $230.8 \pm 2.3$ & $303.2 \pm 3.3$ & $303.0 \pm 1.9$ \\
\hline Beam pipe from $z=0 \mathrm{~cm}$ to end of taper & $397.6 \pm 3.6$ & $499.1 \pm 4.3$ & $428.7 \pm 3.8$ & $338.8 \pm 4.1$ \\
\hline Beam pipe from end of taper & $21.7 \pm 0.9$ & $24.0 \pm 1.0$ & $21.1 \pm 1.0$ & $14.6 \pm 0.8$ \\
\hline Lower shielding vessel for SC $1-3(r<50 \mathrm{~cm})$ & $7.6 \pm 0.2$ & $12.5 \pm 0.3$ & $9.7 \pm 0.2$ & $5.7 \pm 0.2$ \\
\hline Upper shielding vessel for SC $1-3(r>50 \mathrm{~cm})$ & $6.0 \pm 0.1$ & $9.3 \pm 0.2$ & $7.8 \pm 0.2$ & $4.3 \pm 0.1$ \\
\hline Shielding vessel for SC 4-6 & $3.5 \pm 0.3$ & $4.5 \pm 0.3$ & $3.6 \pm 0.3$ & $2.4 \pm 0.2$ \\
\hline Shielding vessel for SC 7-9 & $0.8 \pm 0.1$ & $0.8 \pm 0.1$ & $0.7 \pm 0.1$ & $0.6 \pm 0.1$ \\
\hline Shielding vessel for SC 10-12 & $0.5 \pm 0.1$ & $0.6 \pm 0.1$ & $0.5 \pm 0.1$ & $0.3 \pm 0.1$ \\
\hline Pool reservoir container & $10.5 \pm 0.3$ & $17.1 \pm 0.4$ & $14.0 \pm 0.4$ & \\
\hline Pool reservoir & $460.8 \pm 9.7$ & $814.1 \pm 10.6$ & $655.3 \pm 11.5$ & \\
\hline Jet/target & $416.8 \pm 2.4$ & $167.3 \pm 1.0$ & $298.7 \pm 2.3$ & $1018.5 \pm 5.2$ \\
\hline Be window & $8.9 \pm 0.1$ & $6.3 \pm 0.1$ & $8.4 \pm 0.1$ & $5.1 \pm 0.1$ \\
\hline Total & $3320.7 \pm 14.4$ & $3288.1 \pm 15.0$ & $3323.8 \pm 16.3$ & $3405.6 \pm 11.8$ \\
\hline
\end{tabular}

organic $(\mathrm{MgO})$ insulators, assuming the maximum permitted radiation dose is $10 \mathrm{MGy}$ [15].

About 2.4 MW is deposited in the combined shielding and beam pipe sections. Removing the heat load from these materials will prove quite challenging. The target and mercury pool receives a total power deposition approximately equal to $0.9 \mathrm{MW}$, which must be dissipated in a heat exchanger in the mercury flow return loop.

TABLE III. Power deposition in various regions of the increased shielding geometry configuration for the mercury jet for a range of operational failure modes.

\begin{tabular}{|c|c|c|c|c|}
\hline \multirow[b]{2}{*}{ Region } & \multicolumn{4}{|c|}{ Deposited power $(\mathrm{kW})$} \\
\hline & Nominal & No $\mathrm{Hg}$ jet & No $\underline{B}$ & No $\underline{B}$ and no $\mathrm{Hg}$ jet \\
\hline SC coils $1-12$ & $0.57 \pm 0.05$ & $0.43 \pm 0.05$ & $0.50 \pm 0.05$ & $0.51 \pm 0.05$ \\
\hline Lower shielding SC $1-3(r<50 \mathrm{~cm}, z<83 \mathrm{~cm})$ & $1284.4 \pm 8.3$ & $42.0 \pm 0.3$ & $842.8 \pm 5.8$ & $842.9 \pm 5.5$ \\
\hline Lower shielding SC $1-3(r<50 \mathrm{~cm}, z>83 \mathrm{~cm})$ & $234.2 \pm 3.5$ & $668.3 \pm 2.9$ & $1370.3 \pm 9.4$ & $1371.2 \pm 9.4$ \\
\hline Upper shielding SC 1-3 $(r>50 \mathrm{~cm})$ & $58.3 \pm 0.8$ & $120.7 \pm 0.8$ & $61.6 \pm 0.8$ & $61.7 \pm 0.7$ \\
\hline Shielding for SC 4-6 & $38.0 \pm 1.7$ & $15.0 \pm 1.0$ & $23.8 \pm 1.4$ & $23.9 \pm 1.3$ \\
\hline Shielding for SC 7-9 & $11.0 \pm 0.7$ & $0.8 \pm 0.3$ & $3.1 \pm 0.5$ & $3.0 \pm 0.4$ \\
\hline Shielding for SC $10-12$ & $7.4 \pm 0.7$ & $0.2 \pm 0.1$ & $1.6 \pm 0.3$ & $1.6 \pm 0.4$ \\
\hline Beam pipe up to $z=0 \mathrm{~cm}$ & $352.3 \pm 2.9$ & $0.2 \pm 0.1$ & $0.7 \pm 0.1$ & $0.7 \pm 0.1$ \\
\hline Beam pipe from $z=0 \mathrm{~cm}$ to end of taper & $397.6 \pm 3.6$ & $218.8 \pm 1.7$ & $1013.5 \pm 5.0$ & $1012.8 \pm 5.5$ \\
\hline Beam pipe from end of taper & $21.7 \pm 0.9$ & $0.5 \pm 0.2$ & $4.6 \pm 0.4$ & $4.6 \pm 0.5$ \\
\hline Lower shielding vessel for SC $1-3(r<50 \mathrm{~cm})$ & $7.6 \pm 0.2$ & $12.0 \pm 0.2$ & $8.0 \pm 0.2$ & $8.0 \pm 0.2$ \\
\hline Upper shielding vessel for SC $1-3(r>50 \mathrm{~cm})$ & $6.0 \pm 0.1$ & $11.8 \pm 0.1$ & $6.3 \pm 0.1$ & $6.2 \pm 0.1$ \\
\hline Shielding vessel for SC 4-6 & $3.5 \pm 0.3$ & $1.9 \pm 0.2$ & $2.6 \pm 0.3$ & $2.6 \pm 0.2$ \\
\hline Shielding vessel for SC 7-9 & $0.8 \pm 0.1$ & $0.1 \pm 0.1$ & $0.2 \pm 0.1$ & $0.2 \pm 0.1$ \\
\hline Shielding vessel for SC $10-12$ & $0.5 \pm 0.1$ & $<0.1$ & $0.1 \pm 0.1$ & $0.1 \pm 0.1$ \\
\hline Pool reservoir container & $10.5 \pm 0.3$ & $10.8 \pm 0.1$ & $14.1 \pm 0.2$ & $14.2 \pm 0.2$ \\
\hline Pool reservoir & $460.8 \pm 9.7$ & $2603.2 \pm 4.8$ & $235.9 \pm 2.5$ & $237.5 \pm 2.4$ \\
\hline Jet/target & $416.8 \pm 2.4$ & & $1.9 \pm 0.1$ & \\
\hline Be window & $8.9 \pm 0.1$ & $0.1 \pm 0.1$ & $0.3 \pm 0.1$ & $0.3 \pm 0.1$ \\
\hline Total & $3320.7 \pm 14.4$ & $3707.0 \pm 6.0$ & $3591.9 \pm 12.5$ & $3591.9 \pm 12.6$ \\
\hline
\end{tabular}


The downstream Be-He-Be window (1.5 cm thick) receives a large energy deposition of $9 \mathrm{~kW}$. About $250 \mathrm{~kW}$ will continue into the downstream pion-muon transport system. This will contain scattered, high-energy protons that must be removed by a chicane and absorber system in order to stop radiation damage to other accelerator components [16].

Table II also shows the deposited power in the various regions when alternative materials are used for the target. In general, most of the differences are seen in the energy deposition in the target itself, the shielding, and the liquid/ powder collection pool. Less protons interact with the liquid gallium jet target, going directly into the pool reservoir. This very slightly reduces the overall power deposited in the shielding and beam pipe sections. The reduced density in the tungsten powder jet also means that more energy is deposited in the powder collection reservoir (assumed to have the same geometry as the mercury pool), although not as much as was the case for the gallium target. In contrast, the solid tungsten target itself experiences a much larger energy deposition, corresponding to a quarter of the total beam power. Experimental work using fast, high current pulses passing through tungsten wires has demonstrated that a $20 \mathrm{~cm}$-long, $2 \mathrm{~cm}$ diameter tungsten target will be able to withstand the peak stresses from such an energy deposition, allowing a target lifetime of at least three years [17]. The removal of the reservoir pool for the
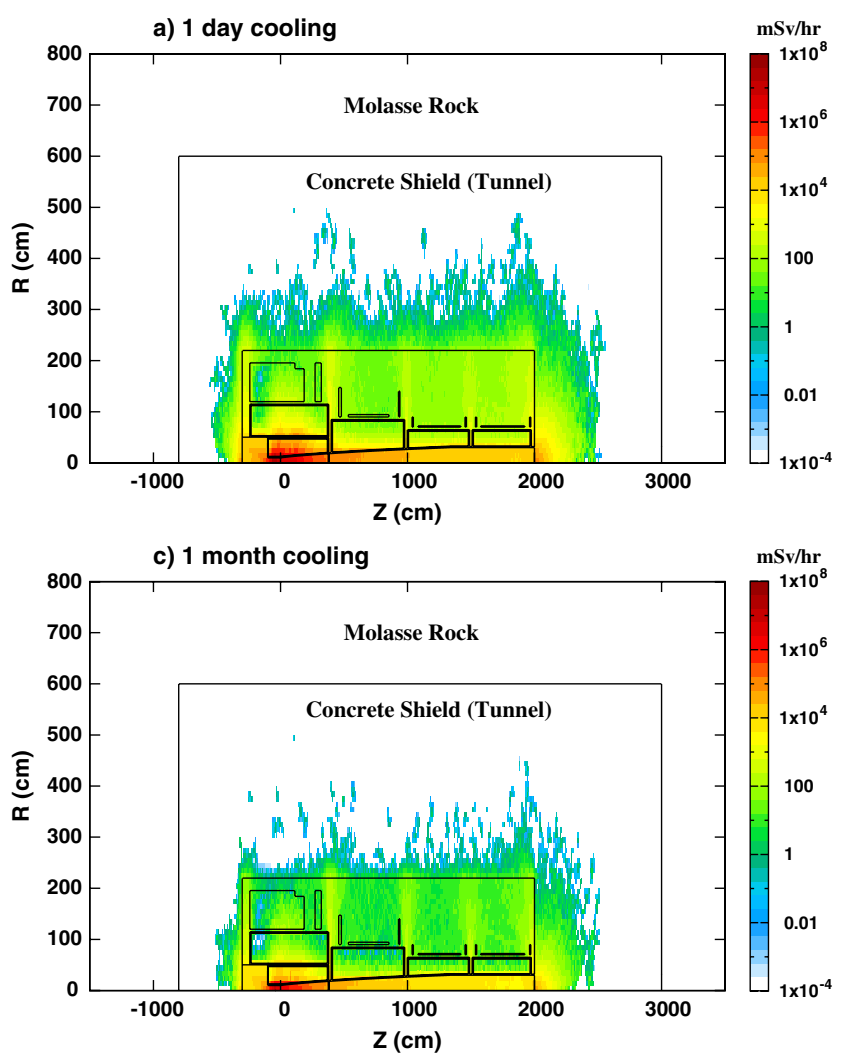

solid target means that about 5\% more energy is deposited in the shielding, with a slight reduction in the radiation dose absorbed by the downstream beam pipe.

\section{FAILURE MODES}

It is important to know how the $4 \mathrm{MW}$ total beam power will be distributed within the new target station geometry for specific (baseline) failure modes: when the magnetic field fails, when the mercury jet stops flowing, and when both happen together. Table III shows comparisons of the average power deposition in various regions of the target station between normal and failure-mode operating scenarios.

Under normal conditions, noninteracting protons from the beam have a trajectory that enters the mercury pool reservoir. This is illustrated by the case when there is no mercury jet target, in which more than half of the total beam power $(2.6 \mathrm{MW})$ is deposited in the pool. This will produce significant agitation of the pool surface with splashes of radial velocities expected to approach $50 \mathrm{~ms}^{-1}$ [18]. In contrast, when there is no magnetic field present to steer the proton beam (and any secondary charged particles), the energy deposition for the combined mercury jet and pool system decreases by roughly a factor of 3 . The proton beam almost completely misses the mercury jet target and hits only part of the mercury pool, instead
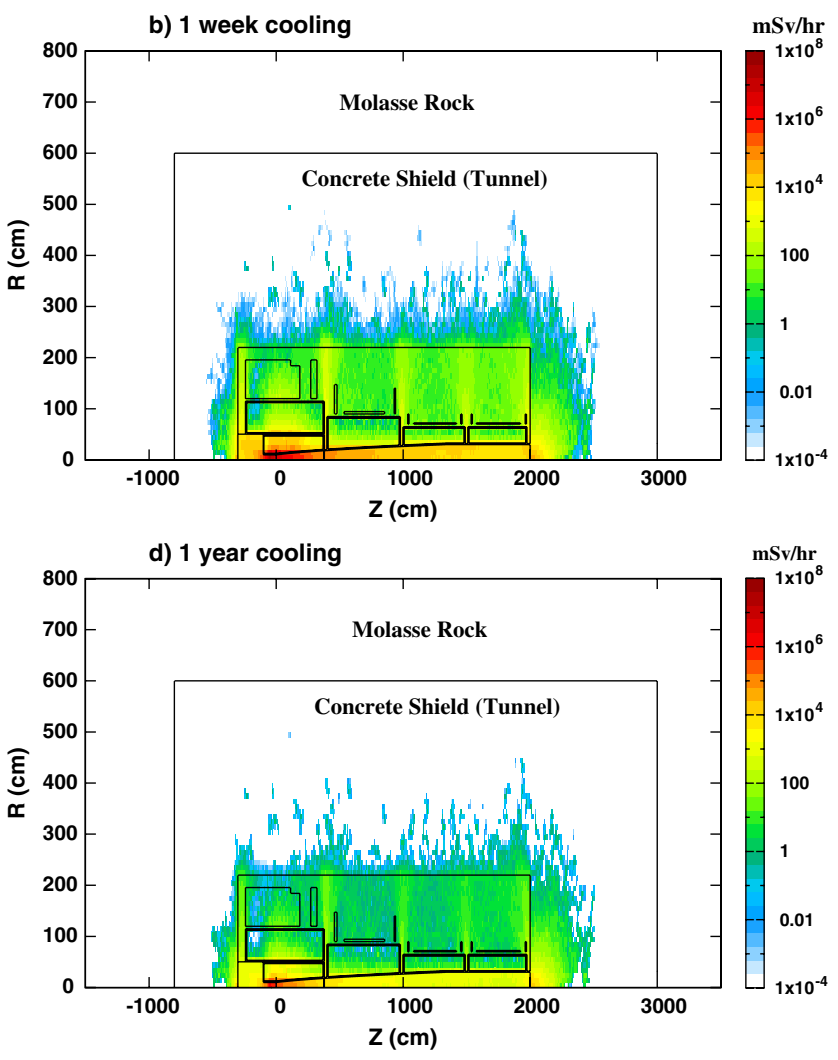

FIG. 8. Ambient dose equivalent rates for the target station, concrete shielding tunnel, and surrounding underground rock. 
dramatically increasing the energy deposition in the surrounding shielding (1.6 to $2.3 \mathrm{MW}$ ) and the nearby beam pipe section (from 0.4 to $1 \mathrm{MW}$ ). For the scenario when there is no mercury jet and no magnetic field, the results are essentially identical to the case when only the magnetic field is turned off, owing to the fact that the proton beam will miss the target in both cases.

\section{RADIATION SAFETY}

Any construction of the target station must take into account the safety requirements of the surrounding environment to prevent radiation contamination of the soil (and ground water). The entire target station must be enclosed within a concrete shielding structure of adequate thickness to stop radiation from escaping; the effective total radiation dose must not exceed $1 \mathrm{mSv}$ per year [19], which is equivalent to a continual residual dose of $0.1 \mu \mathrm{Sv} / \mathrm{hr}$.

Figure 8 shows the estimated total radiation ambient dose equivalent from the $4 \mathrm{MW}$ target station after a total irradiation time of $2 \times 10^{7} \mathrm{~s}$ (1 year), using the FLUKA simulation code and AMB74 conversion factors [20]. The target station is surrounded by a concrete shielding structure (tunnel) that is modeled as three connecting cylindrical sections, extending from $z=-8 \mathrm{~m}$ up to $z=30 \mathrm{~m}$, with an outer radius of $6 \mathrm{~m}$. This tunnel creates a barrier between the surrounding rock, assumed to be molasse soil based at the CERN site, and the radiation generated by the target system. The chemical composition (with mass fractions in parentheses) assumed for the concrete is $\mathrm{O}$ (51.1\%), Si (35.8\%), Ca (8.6\%), Al (2.0\%), Fe (1.2\%), H $(0.6 \%), \mathrm{C}(0.4 \%)$, and $\mathrm{Na}(0.3 \%)$, while the composition of the molasse soil is taken to be O (49.2\%), $\mathrm{Si}(19.8 \%), \mathrm{Ca}$ (9.3\%), $\mathrm{Al}(6.4 \%), \mathrm{C}(4.9 \%), \mathrm{Fe}(4.1 \%), \mathrm{Mg}(3.5 \%), \mathrm{K}$ (1.9\%), Na (0.6\%), Mn (0.2\%), and Ti (0.1\%) [21]. The densities assumed for the concrete and molasse are $2.35 \mathrm{~g} / \mathrm{cc}$ and $2.4 \mathrm{~g} / \mathrm{cc}$, respectively. For a range of cooling decay times, it can be seen that no radiation escapes the concrete shielding, which means that there will be minimal activation of the surrounding soil and ground water. We can also infer that remote handling will be mandatory for maintaining the target system; even at a radial distance of $2 \mathrm{~m}$ from the interaction region, the residual dose rate after 1 year of cooling is of the order of $10 \mathrm{mSv} / \mathrm{hr}$, which greatly exceeds the safety limit for radiation workers (20 mSv/yr) [19].

\section{SUMMARY}

We have presented FLUKA and MARS simulation studies of the pion production and energy deposition in the Neutrino Factory target station. Compared to the original Study 2 geometry, a doubling of the lateral shielding thickness is needed to protect the superconducting coils from radiation. In addition, a reduction in the focusing magnetic field from $20 \mathrm{~T}$ down to $15 \mathrm{~T}$ does not affect the pion production efficiency, provided the inner bore radius is increased. Alternative target materials were also investigated, such as liquid gallium, and powdered and solid tungsten. Each of these offer comparable muon yields, with some differences observed in the power deposited in the target, collection pool, and surrounding shielding. We have investigated what effect various operational failure modes have on the deposited power in the target station. Finally, we have provided estimates of the amount of concrete shielding that will be needed to protect the environment from the high radiation generated by the target station, with remote handling mandatory for any maintenance work.

\section{ACKNOWLEDGMENTS}

We acknowledge the financial support of the European Community under the European Commission Framework Programme 7 Design Study: EUROnu, Project No 212372. We also thank colleagues from the International Design Study (IDS-NF) collaboration for fruitful discussions concerning this work.

[1] J. Pozimski et al., "The Neutrino Factory" (unpublished).

[2] R. J. Abrams et al. (IDS-NF Collaboration), arXiv:1112.2853.

[3] K.T. McDonald et al., in Proceedings of the First International Particle Accelerator Conference, Kyoto, Japan, 2010 (ICR, Kyoto, 2010), ISBN 978-92-9083352-9, p. 3527.

[4] G. Battistoni et al., in Proceedings of the Hadronic Shower Simulation Workshop 2006, AIP Conf. Proc. No. 896 (AIP, New York, 2007), p. 31; A. Fasso, A. Ferrari, J. Ranft, and P.R. Sala, Reports No. CERN2005-10, 2005, No. INFN/TC_05/11, and No. SLAC-R773.

[5] N. V. Mokhov, The MARS Code System User's Guide (Fermilab, Batavia, IL, 1995), Fermilab-FN-628; N. V. Mokhov et al., Recent Enhancements to the MARS15 Code (Fermilab, Batavia, IL, 2004), Fermilab-Conf-04/ 053.

[6] S. Geer and M. Zisman, BNL-Tech Report No. 723692004, 2004.

[7] X. Ding, D. Cline, H. Kirk, and J. S. Berg, in Proceedings of the 23rd Particle Accelerator Conference, Vancouver, Canada, 2009 (TRIUMF, Vancouver, Canada, 2009), p. 2748.

[8] R.C. Fernow, in Proceedings of the 2005 Particle Accelerator Conference, Knoxville, Tennessee, USA (IEEE, Piscataway, NJ, 2005), ISBN 0-7803-8860-7, p. 2651.

[9] J. Strait, N. V. Mokhov, and S. I. Striganov, Phys. Rev. ST Accel. Beams 13, 111001 (2010).

[10] N. Souchlas et al., in Proceedings of the 2011 Particle Accelerator Conference, New York, USA (Brookhaven National Laboratory, New York, 2011), p. 1166.

[11] J. J. Back, JINST 6, P06002 (2011). 
[12] R. J. Weggel et al., in Proceedings of the 2nd International Particle Accelerator Conference, San Sebastián, Spain, 2011 (EPS-AG, Spain, 2011), ISBN 978-92-9083-366-6, p. 1650.

[13] S. A. Maloy, R. S. Lillard, W. F. Sommer, D. P. Butt, F. D. Gac, G. J. Willcutt, and M. R. Louthan, J. Nucl. Mater. 431, 140 (2012).

[14] R. J. Weggel et al., in Proceedings of the 3rd International Particle Accelerator Conference, New Orleans, Louisiana, USA, 2012 (IEEE, Piscataway, NJ, 2012), ISBN 978-395450-115-1, p. 2591.

[15] J. H. Schultz, in Proceedings of the 20th IEEE Symposium on Fusion Engineering (IEEE, Piscataway, NJ, 2003), ISBN 978-0-78037-908-4, p. 423.

[16] C. T. Rogers, D. Neuffer, and P. Snopok, in Proceedings of the 3rd International Particle Accelerator Conference,
New Orleans, Louisiana, USA, 2012 (Ref. [14]), ISBN 978-3-95450-115-1, p. 223.

[17] J. R. J. Bennett, G. P. Škoro, J. Back, S. J. Brooks, T. R. Edgecock, S. A. Gray, A. J. McFarland, K. J. Rodgers, and C. N. Booth, Nucl. Instrum. Methods Phys. Res., Sect. A 646, 1 (2011).

[18] A. Fabich and J. Lettry, Nucl. Instrum. Methods Phys. Res., Sect. A 503, 336 (2003).

[19] CERN Safety Code Manual F (revised), EDMS 335729 (2006).

[20] S. Roesler and G. R. Stevenson, CERN-Tech Report No. SC-2006-070-RP-TN, EDMS 809389, 2006.

[21] H. Vincke and G. R. Stevenson, in Shielding Aspects of Accelerators, Targets and Irradiation Facilities (OECD Nuclear Energy Agency, Paris, 2001), Vol. 5, p. 47. 